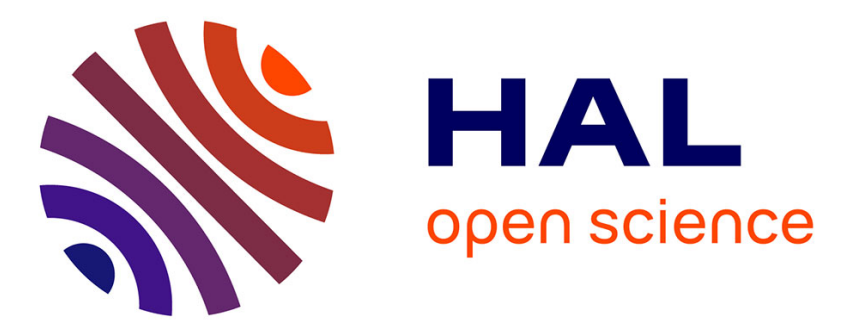

\title{
The protective effect of mindfulness and compassion meditation practices on ageing: hypotheses, models and experimental implementation
}

Antoine Lutz, Gael Chételat, Fabienne Collette, Olga M Klimecki, Natalie L Marchant, Julie Gonneaud

\section{To cite this version:}

Antoine Lutz, Gael Chételat, Fabienne Collette, Olga M Klimecki, Natalie L Marchant, et al.. The protective effect of mindfulness and compassion meditation practices on ageing: hypotheses, models and experimental implementation. Ageing Research Reviews - ARR, 2021. hal-03540925

\section{HAL Id: hal-03540925 \\ https://univ-lyon1.hal.science/hal-03540925}

Submitted on 24 Jan 2022

HAL is a multi-disciplinary open access archive for the deposit and dissemination of scientific research documents, whether they are published or not. The documents may come from teaching and research institutions in France or abroad, or from public or private research centers.
L'archive ouverte pluridisciplinaire HAL, est destinée au dépôt et à la diffusion de documents scientifiques de niveau recherche, publiés ou non, émanant des établissements d'enseignement et de recherche français ou étrangers, des laboratoires publics ou privés. 
The protective effect of mindfulness and compassion meditation practices on ageing: hypotheses, models and experimental implementation

Antoine Lutz ${ }^{\mathrm{a}^{*}}$; Gael Chételat ${ }^{\mathrm{b}^{*}} ;$ Fabienne Collette $^{\mathrm{c}}$; Olga M. Klimecki ${ }^{\mathrm{d}}$; Natalie L. Marchant ${ }^{\mathrm{e}}$; Julie Gonneaud ${ }^{\mathrm{b}}$

Author Affiliations

a Lyon Neuroscience Research Center Inserm U1028, CNRS UMR5292, Lyon 1 University, Lyon, France

b Inserm, Inserm UMR-S U1237, Université de Caen-Normandie, GIP Cyceron, Caen, France

${ }^{c}$ GIGA-CRC In Vivo Imaging, Université de Liège, Liège, Belgium

${ }^{d}$ Institute of Clinical Psychology and Psychotherapy, TU Dresden, Germany

e Division of Psychiatry, University College London, London, United Kingdom

* both authors equally contribute

The authors declare no competing interest.

To be submitted to Ageing Research Reviews

Keywords: Alzheimer's disease; Anxiety; Cognition; eudaimonic well-being; MeditAgeing; Silver Sante Study 
Corresponding authors: Antoine Lutz, antoine.lutz@inserm.fr and Gaël Chételat, chetelat@cyceron.fr

\section{Abstract:(200 words max, now 219)}

Alzheimer's disease $(A D)$ represents a major health and societal issue; there is no treatment to date and the pathophysiological mechanisms underlying this disease are not well understood. Yet, there is hope that $A D$ risk factors and thus the number of $A D$ cases can be significantly reduced by prevention measures based on lifestyle modifications as targeted by non-pharmacological preventive interventions. So far, these interventions have rarely targeted the psycho-affective risk factors related to depression, stress, anxiety, and feeling of loneliness, which are all prevalent in ageing. This paper presents the hypothesis that the regular practice of mindfulness meditation (MM) and loving-kindness and compassion meditation (LKCM) in the ageing population constitutes a lifestyle that is protective against $A D$. In this model, these practices can promote cognition, mental health, and well-being by strengthening attention control, metacognitive monitoring, emotion regulation and pro-social capacities. Training these capacities could reduce the risk of $A D$ by upregulating beneficial age-related factors such as cognitive reserve, and down-regulating detrimental age-related factors, such as stress, or depression. As an illustration, we present the Medit-Ageing study (public name Silver Santé Study), an on-going European project that assesses the impact and 
mechanisms of non-pharmacological interventions including meditation, in the ageing population.

\section{Objectives:}

Our main objective is to propose a model that outlines the cumulative impact that practices of mindfulness meditation ( $\mathrm{MM}$ ) and loving-kindness and compassion meditation (LKCM) (Table 1) could have on cognition, mental health, lifestyle, and AD risk in the ageing population. Drawing on previous research with younger adults, the proposed model aims to function as a heuristic tool to illustrate the putative cognitive, neuroscientific and biological mechanisms of meditation training in ageing. We hope that this framework will stimulate and structure research in this area to support the integration of meditation in non-pharmacological preventive approaches against $A D$.

\section{Background:}

\section{a. Historical evolution}

Over the last 20 years, evidence has accumulated towards demonstrating the significant influence of environmental factors, including modifiable lifestyle risk factors, on the development of Alzheimer's disease (Norton et al., 2014; Yegambaram et al., 2015).

The main modifiable risk factors identified to date include low education, smoking, physical inactivity, depression, mid-life hypertension, diabetes and mid-life obesity (Norton et al., 2014). The most relevant in late life are smoking, depression, physical 
inactivity, social isolation, and diabetes (Montero-Odasso et al., 2020). There is also evidence for other potentially modifiable risk factors in older adults, including hearing loss, diet, anxiety, neuroticism, rumination, repetitive negative thinking and sleep disorders(Marchant et al., 2020; Marchant and Howard, n.d.; Montero-Odasso et al., 2020; Zufferey et al., 2017). Studies showed that 30 to $40 \%$ of dementia cases - of which $A D$ is the primary cause - are due to modifiable risk factors (Montero-Odasso et al., 2020; Norton et al., 2014). This suggests that a reduction of modifiable risk factors, and possibly the promotion of protective factors, also constitutes a powerful lever towards primary and secondary prevention and reduction of AD cases. Estimation of the possible impact of interventions designed to modify these risk factors are very encouraging, showing that a reduction of $20 \%$ per decade in the prevalence of the main risk factors might reduce the number of $A D$ cases in 2050 by $15.3 \%$ worldwide, i.e. 16.2 million fewer cases (Norton et al., 2014). Multi-target pharmacological trials and lifestyle-based non-pharmacological interventions (and possibly the combination of both) thus represent our best hope to target several of the possible pathways of the disease. Even when a disease-modifying treatment becomes available, the mechanistic understanding of prevention-based reduction of AD risk factors will still be needed.

\section{b. Rationale}

The concept of reserve was the first step towards integrating our knowledge on the impact of lifestyle on the physiopathology of the disease (Scarmeas and Stern, 2003). This concept accounts for individual differences in susceptibility to age-related brain changes or AD-related pathology. It proposes notably that a set of life experiences, 
such as higher educational or occupational attainment, allows some people to cope better than others with brain damage. This concept has stimulated research since the 1990s, and has progressively broadened to include other aspects of life experience such as physical activity, regulation of vascular risk factors, diet, and social stimulation.

Following this line of evidence, non-pharmacological lifestyle-based clinical trials were designed to target one or several risk factors simultaneously. Thus, the three largest multi-domain randomized controlled trials to date (FINGER, PreDIVA and MAPT) (Carrié et al., 2012; Kivipelto et al., 2013; Richard et al., 2009) incorporated several of the following interventions simultaneously: nutritional guidance, management of metabolic and vascular risk factors, physical exercise, social activities, and cognitive training (Carrié et al., 2012; Kivipelto et al., 2013; Richard et al., 2009). Findings from the FINGER study were particularly encouraging, showing for instance a positive effect of the multi-domain intervention on cognitive functions, and on mitigating the dementia risk as measured by an improvement on the "LIfestyle for BRAin Health" (LIBRA) score (Ngandu et al., 2015; Deckers et al., 2021). These demonstrated proof-of-concepts led to the expansion of the project into a worldwide consortium, called World Wide FINGER. This consortium includes the U.S. POINTER (https://alz.org/us-pointer/), which is a replication study in the US. Noticeably, these interventions targeted all the main risk factors described above with the exception of psycho-affective risk factors, including depression, anxiety, neuroticism, rumination, repetitive negative thinking, which are the focus of the present model.

4. New or updated hypothesis

a. Meditation and ageing: early observational data 
We and others have already hypothesized that the sustained and regular practice of mindfulness meditation ( $\mathrm{MM}$ ) can positively affect ageing by improving mental health and well-being and by reducing AD risk ((Acevedo et al., 2016; Chételat et al., 2018; Fountain-Zaragoza and Prakash, 2017; Innes and Selfe, 2014; Kurth et al., 2017; Malinowski and Shalamanova, 2017)). MM primarily develops attention regulation, and meta-cognitive and interoceptive capacities to monitor and regulate emotions, particularly by deconstructing negative automatic mental schemes and fostering emotional balance (e.g. (Dahl et al., 2015; Lutz et al., 2008), see Figure 1 and Table 1). MM can foster lifestyle medicine and health behavior changes by strengthening 1) awareness of unhealthy behaviors, 2) cognitive control of automatic stress reactions that reinforce habits (e.g., inactivity, overeating), and 3) the ability to make healthier choices and sustain behavior change (Schuman-Olivier et al., 2020; Victorson et al., 2015). Using MM-based intervention is acceptable, feasible, and in-demand for older adults with or without cognitive decline (Berk et al., 2017; Geiger et al., 2016; Lenze et al., 2014). MM-related attention training is thought to exhibit high transfer capacity to other attentional cognitive processes and daily situations, making it a promising technique for $A D$ prevention programs (Malinowski and Shalamanova, 2017). In these proposals, MM is typically thought to impact ageing by strengthening attentional processes, positive affects, psychological well-being and sleep quality, while downregulating risk factors such as stress, inflammation and mood disorders (Figure 2). For some authors, the slowing down of age-related cognitive decline would be particularly relevant at earlier life stages as prevention while the down-reduction of psycho-affective risk factors could be prioritized at a later life stage (Figure 2, (Berk et 
al., 2017). Evidence for these claims are largely based on observational and intervention-based meditation research in young and middle-aged adults. More specifically, these studies show that MM practice improves cognition (mainly attention, but also memory), reduces sleep disorders (including insomnia), stress, anxiety, depression, feelings of loneliness and social exclusion, and cardiovascular risk factors (Dimidjian and Segal, 2015; Donald et al., 2019; Kim et al., 2017; Lutz et al., 2015; Tang et al., 2015). Moreover, the effects of meditation on brain structure and function have consistently been reported in young and middle-aged adults, especially in frontal and limbic structures, as well as in the insula (Fox et al., 2016, 2014, p. 201) (Figure 3). Interestingly, these structures are known to be particularly sensitive to ageing and AD. MM training has also been associated with increased telomerase activity in blood cells (Schutte and Malouff, 2014), and reduced molecular, genetic and epigenetic markers of inflammation(Chaix et al., 2017; Kaliman et al., 2014). Preliminary evidence also comes from cross-sectional studies in expert meditators. While reflecting a life-long meditation experience and subject to multiple biases related to the cross-sectional nature of these kinds of studies, findings in elderly expert meditators support the hypothesis that meditation can preserve brain structure and glucose metabolism (e.g. (Chételat et al., 2017; Luders et al., 2016)). We showed for instance preserved grey matter volume and/or more glucose metabolism in six older-adult expert meditators compared to 67 age-matched controls in brain regions sensitive to ageing, including ventromedial prefrontal cortex, anterior cingulate cortex, bilateral temporo-parietal junction, insula and posterior cingulate cortex (Chételat et al., 2017). Interestingly, most of these regions also showed the strongest age-related decrease from 20 to 87 years in a cohort of 186 
controls (Chételat et al., 2017), and the temporo-parietal and posterior cingulate cortex are known as being the most sensitive brain regions to early AD-related glucose metabolism changes (Teipel et al., 2015). In support of a preventive effect of meditation on cognitive decline in ageing, cross-sectional studies have also found that elderly longterm meditators have higher levels of attention, executive function, and fluid intelligence compared with non-meditators (Gard et al., 2014; Prakash et al., 2012).

While longitudinal research on meditation in the elderly population is still in its infancy, novel interventions, such as the Mindfulness-Based Cognitive Approach for Seniors (MBCAS), have been tailored to the needs of older adults. Like other mindfulness-based programs, the MBCAS program aims to teach older adults to monitor without judgement and to positively cope with their habitual responses and reactions to various emotions and stressors. Yet, this program has specifically adapted the mindfulness exercises to the physical and psychosocial challenges related to aging (Zellner Keller et al., 2014). There is emerging evidence indicating that mindfulness-based interventions can impact perceived well-being, executive functioning and memory in healthy ageing (Moynihan et al., 2013), and in patients at risk or with AD (Quintana-Hernández et al., 2015; Smart et al., 2016). One longitudinal study assessing the impact of eight weeks of mindfulnessbased meditation on brain connectivity found that meditation versus relaxation training decreased network connectivity within the default mode network in older adults, which may indicate more efficient cognitive processes (Cotier et al., 2017). While these findings are encouraging, further randomized control trials with large samples are 
necessary to confirm that $\mathrm{MM}$ training improves cognition and emotion regulation in older adults, and has therefore the potential to promote healthy ageing.

b. The "Medit-Ageing" hypothesis, and the SCD-Well and Age-Well randomized controlled trials

We further refine and update these early proposals on MM and ageing in two ways. Firstly, we propose to integrate LKCM in the model. Compared to MM, LKCM is thought to primarily engage perspective taking, empathy and cognitive reappraisal to strengthen motivational processes and to stimulate and reinforce automatic positive schemes involved in caring and pro-social behaviors (Dahl et al., 2015; Singer and Klimecki, 2014) (Figure 1, Table 1). We argue that the combination of both MM and LKCM within a single intervention could optimize the transformative effects of meditation interventions on ageing. Secondly, we advocate for the need to investigate interventions across a longer timescale than the standard 8-week meditation intervention in order to characterize effects on the biology of ageing. We finally illustrate this model with the Medit-Ageing study, an ongoing European study of meditation and ageing.

Our proposal to integrate and combine LKCM with MM is consistent with traditional models of meditation training, and of contemporary LKCM-based interventions, which often start with MM before introducing LKCM (Singer and Klimecki, 2014). It is also congruent with emerging clinical evidence showing a complementary and beneficial role of LKCM-based interventions on anxiety and depression, positive emotions, purpose in life and social support (Donald et al., 2019; Fredrickson et al., 2008; Graser and 
Stangier, 2018). The training in emotional balance is particularly relevant for ageing as purpose in life and personal growth usually decrease with ageing, and high levels of purposeful life engagement predict extended longevity, reduced physiological dysregulation, and gene expression linked to better inflammatory profiles (Ryff et al., 2016, p. 201). In addition, while both LKCM and MM strengthen the capacities to embrace stressors (see Table 1), the emphasis of the others' care and wellness in LKCM training could be particularly relevant to cope with the dyadic stressors for instance between a person diagnosed with a young-onset dementias and a spousal caregiver (see Table 1 and (Bannon et al., 2021)). While there is evidence that MM-based interventions train implicitly some processes related to LKCM (Donald et al., 2019), some recent evidence from affective neurosciences argue for supplementing these programs with an explicit training in LKCM. Consistently, training in LKCM and MM within the same participants induces different structural and functional neuroplastic changes (Klimecki et al., 2013; Valk et al., 2017), suggesting different brain mechanisms and, therefore, possibly complementary effects (Figure 3). For instance, LKCM specifically modulates brain regions such as the striatum and medial orbitofrontal cortex during the perception of emotional stimuli (Klimecki et al., 2013). LKCM also plays a distinct role in reducing social stress and stress reactivity compared to MM (Engert et al., 2017), suggesting an alternative pathway to regulate stress. However, while clinical interventions promoting more broadly positive psychology in older adults have reported encouraging findings (Friedman et al., 2019), this mechanistic evidence on LKCM is currently only based on young and middle aged adults. 
Extending previous proposals on MM, Figure 1 summarizes the complementary but also overlapping cognitive mechanisms likely engaged in MM and LKCM. In the present framework, the common purpose of these practices is to reinforce positive psychological functioning and eudaimonic well-being (Dahl et al., 2015). The common overlapping mechanisms include attention control and meta-awareness. In addition, MM specifically down-regulates maladaptive patterns of thinking or emotional reactivity involved in the escalation of negative mood by learning to engage flexibly with different interpretations of the mental events (see Table 1 for an illustrative example of these concepts and (Lutz et al., 2015) for review). By contrast, LKCM specifically upregulates positive emotions by explicitly cultivating caring, benevolence and pro-social habits. Training these capacities would recruit underlying brain structure and function, especially in frontal and limbic areas (Dahl et al., 2015; Fox et al., 2016; Singer and Klimecki, 2014) (see Figure 3). It would also downregulate brain network interactions, connectivity patterns and structural changes previously linked to maladaptive emotional states such as chronic stress and depression (e.g. on amygdala responsivity (Kral et al., 2018)). Finally, we hypothesize that these psychological, cognitive, behavioral and brain changes can strengthen protective factors and downregulate detrimental factors of ageing (Figure 2). The specific modulatory effects of MM and LKCM on these processes are not currently known. The positive and negative factors listed in Figure 2 likely exhibit recursive relationships between psychological and biological factors at multiple levels (genes, cells, neural circuits, physiology), which would span over multiple time-scales. 
For this reason, in the second aspect of our proposal, we further advocate for the need to investigate meditation interventions at multiple time-scales, spanning across several years, to detect the modulatory effect of meditation on the biological, behavioral and neural markers of ageing. The field of meditation research has almost exclusively focused on short 8-week interventions (with rare exceptions, such as the ReSource project, proposing a 9-month meditation intervention (Valk et al., 2017)). Little is known about the chronometry or time scale of responses to meditation practice, but there is some evidence that some of these effects require sufficient amounts of training to be noticeable. For instance, less amygdala reactivity has been reported in experienced meditators while no such reduction was observed in participants who received a standard 8-week MM-based intervention (Kral et al., 2018). Theoretical models of meditation practices predict an "upward spiral" trajectory of development, where there is a mutual reinforcement between various protective factors of well-being (Barnhofer, 2019; Garland et al., 2017). Such a dynamic perspective on meditation training predicts both an accumulating effect of meditation practice, but also some non-linear interactions among these various factors. Sampling multiple time scales is critical to describe the complex dynamic of these psychological, neural and biological changes in response to meditation practice. Here we specifically hypothesize a cascading effect starting by meditation-related changes in psychological processes, which could then impact affective and cognitive risk factors, then biological risk factors, and then gradually altering ageing-related processes (Figure 2). 
The present model and proposals have guided the design of the Medit-Ageing study (public name: Silver Santé Study; www.silversantestudy.eu) which is a European research project focusing on mental health and well-being in ageing populations. Overall, the Medit-Ageing study aims to test the hypothesis that the regular practice of both $\mathrm{MM}$ and LKCM in older adults constitutes a protective lifestyle, not only against $A D$, but also more generally by promoting cognition, mental health, emotional balance, and well-being. It includes two independent clinical studies: SCD-Well and Age-Well. Both constitute a randomized control trial (RCT, ClinicalTrials.gov Identifier: NCT03005652 and NCT02977819), for details see the protocol papers (Poisnel et al., 2018) respectively), while Age-Well additionally includes an observational crosssectional study in older expert long-term meditators (ClinicalTrials.gov Identifier: NCT02977819, (Lutz et al., 2018)). Both interventions used a MM-based intervention that was specifically tailored to the needs of older adults building on modifications suggested by Zellner Keller et al., 2014 together with a particular focus on LKCM. The SCD-Well intervention lasts 8 weeks (2 months) and targets patients suffering from subjective cognitive decline (SCD) where elderly patients experience concerns about cognitive functioning that generates significant emotional difficulties as reflected in persisting symptoms of anxiety and depression. The Age-Well intervention lasts 18 months and targets cognitively unimpaired adults older than 65 years, who have an interest in learning meditation or a foreign language. These two RCTs investigate meditation-related changes at multiple time-scales: for SCD-Well at t0+8 weeks (postintervention) and t0+6 months ( 4 months post-intervention), and for Age-well at t0+9 months, t0+18 months (post-intervention) and t0+44 months (26 months post- 
intervention). The meditation interventions aim to train attention and improve cognition, countering stress and improving emotional well-being, and increasing positive psychology, social connectedness and countering feelings of loneliness (Figures 1-2 and Table 1). Our cascading hypothesis (see above) predicts that the 8-week SCD-Well intervention should primarily impact the regulation of specific psycho-affective and cognitive processes, with change in anxiety symptoms as the primary study outcome. Its aim could be understood as supporting or building resilience, that is to develop patients' skills to cope with stress and adversity, in particular due to concern about cognitive functioning. By contrast the 18-month Age-Well intervention should additionally impact biological and neural processes and the regulation of specific lifestyles, including those associated with $A D$ risk, that would eventually impact protracted ageing processes. Beyond developing resilience, this longer intervention could also develop resistance by inducing long-lasting biological protective effects. Its primary outcomes are objective biomarkers of brain integrity in the anterior insula and cingulate cortices.

The cross-sectional study in experts explores the effect of lifelong meditation practice. Altogether the Medit-Ageing study provides an opportunity to test the present framework.

\section{Major challenges for the hypothesis}

One challenge will remain to test the hypothesis of an added value of combining MM and LKCM compared to MM alone will be to properly power such study. The effect of interindividual variation in the response to these meditation techniques is not currently 
known, even if preliminary evidence suggests that the responses to MM or LKCM practice can interact with individual characteristics (Barnhofer et al., 2010). Another challenge concerns the generalizability of the empirical data collected during long meditation interventions. While the absence of attrition in the Age-Well study demonstrates the feasibility of this approach, the demands and the commitments required to take part in such studies are likely to bias the demographic of the sample of participants. More generally, the participants' education, social and religious background is expected to modulate the adherence to the therapeutic rationale of meditation.

6. Conclusive remarks: Here we introduce a novel model to account for the putative role of meditation training in reducing AD-related risk. We argue that secular meditationbased interventions constitute a promising complementary approach to other preventive strategies by specifically targeting affective risk factors which are known to be impacted by mental training akin to meditation. More specifically, we propose that combining MM and LKCM training during short and long interventions would optimally impact the relevant age- and AD-related factors. The Medit-Ageing study will allow us to start testing this framework. If positive, the long-term goal of this research project will be to provide an evidence-based program containing its active components. Such interventions could be made available to large-scale audiences of older adults as a stand-alone program, or combined with existing multidomain interventions.

\section{Table 1:}




\section{Definition of meditations:}

Meditation: Meditation can be understood as a family of complex forms of cognitive and affective mental training cultivated for various ends, in particular to decrease human suffering and to increase well-being and human flourishing. We will focus on mindfulness meditation (MM) and loving-kindness and compassion meditation (LKCM), but other meditation techniques can be studied in relation to ageing. Both practices develop positive functioning and eudaimonic well-being, by opposition to hedonic well-being. Positive functioning includes purposeful engagement in life, realization of personal aptitudes and capacities, and enlightened self-knowledge (Ryff et al., 2016). Eudaimonic well-being emphasizes meaning, human potential and self-realization and is defined in terms of the degree to which a person is fully functioning (Ryff et al., 2016). Hedonic well-being is defined in terms of pleasure attainment and pain avoidance. The meditation interventions discussed here are secular.

Mindfulness meditation (MM): Mindfulness, or attentive presence, consists of cultivating a vigilant awareness of one's own thoughts, actions, emotions and motivations. The participant learns to intentionally pay attention to his/her internal or external experiences in the present moment without identifying, being absorbed by these experiences or making any value judgment about them. The aim is that the present moment is lived in a more open and flexible way while being less dominated by mental conditioning that is a source of suffering.

Loving-kindness and compassion meditation (LKCM): The practice of LKCM aims to cultivate feelings of love or compassion for oneself and others and to be more accepting of emotions such as shame, self-criticism, or anger. Building on the non-judgmental monitoring capacity developed in $\mathrm{MM}$, participants learn to cultivate acceptance and kindness toward oneself, for instance in relation to one's negative thoughts, distractions, difficult emotions, unpleasant physical sensations, and to foster appreciation toward positive qualities of one's mind (joy, contentment, ...). The participants can then learn to extend an attitude of care a nd loving-kindness toward their loved ones, toward neutral persons (e.g. strangers), or toward difficult persons, ultimately recognizing that the need for comfort, security, and happiness is shared by all living beings.

Phenomenological illustration of the Medit-Ageing model: To illustrate the cognitive mechanisms likely engaged in MM and LKCM, let us consider a rumination which arises in the mind of an older adult. It might come with thoughts such as "I feel alone and worthless". When the thought arises and one identifies with it, it can appear to be an accurate description of oneself such that a depressed mood is enhanced or sustained. This affective reactivity further ignites the next negative thought. 
Attentional mechanism: To regulate this rumination, the first common mechanism of MM and LKCM is attention regulation and monitoring (Figure 4) which would consist here of becoming aware of one's dementia worry or fear of cognitive decline. Instead of being lost in the thought or emotion, the practitioner can acknowledge the thought or emotion and the associated bodily sensations. Cultivating such nonreactive observations or discernments provide knowledge about the objects of experience and experience itself, which can then be used in the next step of the meditation.

In the Age-Well meditation intervention, this mechanism is mainly trained with MM-related techniques such as Focused Attention meditation on bodily sensations (e.g., breathing exercises, body scan, walking meditation, laying meditation), on external sensations (e.g., to sounds, visual object, food odors, tastes), on feeling tone and emotions (e.g., during physical pain, fatigue, or the loss of a close one), or on thoughts and with Open Monitoring meditation on spontaneous mental activities (e.g. choiceless awareness meditation). This mechanism is implicitly trained in LKCM while one is focusing one's attention for instance on a loved-one while wishing her/him well.

Deconstructive mechanism (Dahl et al., 2015): A second core capacity trained in $\mathrm{MM}$ is to phenomenally interpret thoughts, feelings, and perceptions just as mental processes rather than as accurate depictions of reality. This mechanism is known as 'dereification', or 'cognitive defusion', or 'decentering' (for a discussion on the distinction between these constructs see (Dahl et al., 2015)). This process allows the practitioner to relate flexibly to thoughts without reifying any particular interpretation as the only solution and without feeling emotionally attached or reactive to one particular interpretation (Figure 4, right). At the highest form of dereification, thoughts lose their representational status and are experienced as mere mental events, situated and embodied within a field of sensory, proprioceptive, affective, and somatic feeling tones (Lutz et al., 2015).

In the Age-Well meditation intervention, this mechanism is mainly cultivated with MM-related practices through the cultivation of insights on the automaticity of mind-wandering, on the transient and composite nature of the ageing body, and of thoughts and emotions, on the mental tendency to generalize, time-travel, cognitively fused or reify the contents of experience. These various themes are presented in relation to uncertainties of aging, finitude of life, changes in time perception, and roles/responsibilities during aging, and coping with loss in formal meditation and daily life.

Constructive mechanism: LKCM additionally involves altering the content of thoughts and emotions as opposed to simply observing them in MM. To this end, LKCM uses various reappraisal and perspective taking strategies (Dahl et al., 2015). For instance, and in response to the rumination, the practitioner can generate a feeling of altruistic love (wishing well-being) or of compassion (wishing freedom from suffering) toward oneself and others. He/She can also imagine that, by experiencing this particular rumination, his/her experience will release all beings from this particular feeling of being alone and worthless. The reappraising 
of a negative experience into a meaningful and caring one, can increase positive functioning and decrease maladaptive self-schema (Dahl et al., 2015).

In the Age-Well meditation intervention, this mechanism is mainly trained with LKCM-related exercises such as the cultivation of acceptation, appreciation and loving-kindness toward one's mind-wandering episodes, one's physical pain, one's ageing body, one's thoughts and emotions; LKCM is then cultivated for one-self, for a loved one, for a neutral person, a difficult person and for all beings. Again, these exercises are integrated in daily life and in particular with the personal and dyadic challenges of ageing (Bannon et al., 2021).

\section{Acknowledgments:}

We thank two anonymous reviewers who helped to improve this manuscript. The MeditAgeing project is funded through the European Union in the Horizon 2020 program related to the call PHC22 "Promoting mental well-being in the ageing population." The funder is not involved in the study design, data acquisition, data analysis, data interpretation, or manuscript writing. We thank additional funding from Fondation MMA des Entrepreneurs du futur and the Normandie Région.

Figure 1: Psychological and cognitive mechanisms of $M M$ and LKC meditation practices: MM cultivates the non-reactive monitoring of one's actions, emotions, and thoughts and the flexible regulation of attention and emotions; LKCM cultivates benevolence and care for oneself and for others (see Table 1). The pathway on the right downregulates repetitive negative thinking and afflictive emotions, the one on the left upregulates positive and pro-social states. The states cultivated during these meditation practices influences automatic processes involved in emotional appraisal and autonomic and immune systems.

Figure 2: Mechanisms of action of MM and LKCM meditations on ageing factors: Box 1 illustrates how these meditation practices train particular brain states, which are in turn gradually training specific brain networks (see Figure 3, adapted from (Malinowski and Shalamanova, 2017)). These states and trained networks facilitate the monitoring, 
regulating and updating of automatic afflictive mental patterns, and as such can reduce age-related suffering and enhance resilience and well-being. The box 2 illustrates how the processes could then impact lifestyle factors important in ageing including both upregulation of positive factors (e.g. cognitive and brain reserve, cognitive stimulations, social interactions, well-being, telomere lengths) and down-regulation of negative factors (e.g. cardio-vascular risk factors, depression, chronic stress, anxiety, sleep disturbances, cognitive decline, dementia, frailty, disability). We specifically hypothesize a cascading effect starting by meditation-related changes in psychological processes, which could then impact affective and cognitive risk factors, then biological risk factors, and then gradually altering ageing-related processes. Such a cascading effect across multiple domains and time scales could lead to upward or downward spiral trajectories of ageing. Characterizing the time course of this cascading effect will be important to optimally implement MM-and-LKCM-based interventions for prevention and mental health.

Figure 3: Schematic view of some of the regions affected by ageing (areas with dashed lines), or recruited or impacted by MM (blue), LKCM (yellow) or both (green). Neuroimaging studies can track age-related macroscopic, structural, functional and molecular brain changes. They have shown substantial decreases with age in cerebral volume and glucose metabolism (Chételat et al., 2017; Fjell and Walhovd, 2010). These changes are not homogeneous throughout the brain as they predominate in the frontal cortex $(1,2)$ and are also often reported in the anterior cingulate cortex $(2,3)$, insula (4), sensorimotor, and perisylvian regions (Chételat et al., 2017; Fjell and Walhovd, 2010). Other parietal $(5,6)$ and temporal $(7,8)$ brain regions, including the hippocampus $(8)$, seem to be involved as well, yet findings are less consistent across studies. Findings on MM and LKCM meditation are based on functional or anatomical neuroimaging studies investigating either MM or LKCM states or the plasticity they induced. The withinsubject functional comparison between MM and LKCM is still largely missing. The most consistent brain regions identified across both practices are the mid and anterior cingulate cortex (3), insula (4), precuneus/posterior cingulate cortex (6), amygdala (9) and prefrontal regions in particular, in functional studies, the orbitofrontal and frontopolar cortex (10) (Chételat et al., 2017; Dahl et al., 2015; Fox et al., 2014; Klimecki et al., 2013; Lutz et al., 2008; Valk et al., 2017). While hippocampus (8) is found in MM literature (Chételat et al., 2017; Dahl et al., 2015; Fox et al., 2014; Klimecki et al., 2013; Lutz et al., 2008; Valk et al., 2017) and parahippocampus (11) in LKCM literature (Chételat et al., 2017; Dahl et al., 2015; Fox et al., 2014; Klimecki et al., 2013; Lutz et al., 2008; Valk et al., 2017), the unique role of each practice will need further confirmation. These findings are interpreted in relation to their roles of these areas in emotion and attention regulation, interoception and various socio-affective processing (Chételat et al., 2017; Dahl et al., 2015; Fox et al., 2014; Klimecki et al., 2013; Lutz et al., 2008; Valk et al., 2017). Only a few studies directly compared the impact of MM and LKCM on the brain anatomy. One landmark study showed that MM practice specifically thickened the medial PFC (2, in blue), consistent with its role in metacognition, while the practice of LKCM specifically thickened post and precentral 
gyri (12), inferior frontal gyrus (13) and supramarginal gyri (5) (in yellow), consistent with their role in self/other perspective (Figure created with BioRender.com).

\section{Bibliography:}

Acevedo, B.P., Pospos, S., Lavretsky, H., 2016. The Neural Mechanisms of Meditative Practices: Novel Approaches for Healthy Aging. Curr Behav Neurosci Rep 3, 328-339. https://doi.org/10.1007/s40473-016-0098-x

Bannon, S.M., Grunberg, V.A., Reichman, M., Popok, P.J., Traeger, L., Dickerson, B.C., Vranceanu, A.-M., 2021. Thematic Analysis of Dyadic Coping in Couples With YoungOnset Dementia. JAMA Netw Open 4, e216111. https://doi.org/10.1001/jamanetworkopen.2021.6111

Barnhofer, T., 2019. Mindfulness training in the treatment of persistent depression: can it help to reverse maladaptive plasticity? Current Opinion in Psychology, Mindfulness 28, 262267. https://doi.org/10.1016/j.copsyc.2019.02.007

Barnhofer, T., Chittka, T., Nightingale, H., Visser, C., Crane, C., 2010. State Effects of Two Forms of Meditation on Prefrontal EEG Asymmetry in Previously Depressed Individuals. Mindfulness (N Y) 1, 21-27. https://doi.org/10.1007/s12671-010-0004-7

Berk, L., van Boxtel, M., van Os, J., 2017. Can mindfulness-based interventions influence cognitive functioning in older adults? A review and considerations for future research. Aging \& Mental Health 21, 1113-1120. https://doi.org/10.1080/13607863.2016.1247423

Carrié, I., van Kan, G.A., Gillette-Guyonnet, S., Andrieu, S., Dartigues, J.-F., Touchon, J., Dantoine, T., Rouaud, O., Bonnefoy, M., Robert, P., Cuffi, M.-N., Bories, L., Bordes, S., Gasnier, Y., Desclaux, F., Sudres, K., Pesce, A., Vellas, B., 2012. Recruitment strategies for preventive trials. The MAPT study (MultiDomain Alzheimer Preventive Trial). J Nutr Health Aging 16, 355-359. https://doi.org/10.1007/s12603-012-0046-8

Chaix, R., Alvarez-López, M.J., Fagny, M., Lemee, L., Regnault, B., Davidson, R.J., Lutz, A., Kaliman, P., 2017. Epigenetic clock analysis in long-term meditators.

Psychoneuroendocrinology 85, 210-214. https://doi.org/10.1016/j.psyneuen.2017.08.016

Chételat, G., Lutz, A., Arenaza-Urquijo, E., Collette, F., Klimecki, O., Marchant, N., 2018. Why could meditation practice help promote mental health and well-being in aging? Alzheimer's Research \& Therapy 10. https://doi.org/10.1186/s13195-018-0388-5

Chételat, G., Mézenge, F., Tomadesso, C., Landeau, B., Arenaza-Urquijo, E., Rauchs, G., André, C., de Flores, R., Egret, S., Gonneaud, J., Poisnel, G., Chocat, A., Quillard, A., Desgranges, B., Bloch, J.-G., Ricard, M., Lutz, A., 2017. Reduced age-associated brain changes in expert meditators: a multimodal neuroimaging pilot study. Scientific Reports 7. https://doi.org/10.1038/s41598-017-07764-x

Cotier, F.A., Zhang, R., Lee, T.M.C., 2017. A longitudinal study of the effect of short-term meditation training on functional network organization of the aging brain. Sci Rep 7, 598. https://doi.org/10.1038/s41598-017-00678-8

Dahl, C.J., Lutz, A., Davidson, R.J., 2015. Reconstructing and deconstructing the self: cognitive mechanisms in meditation practice. Trends in Cognitive Sciences 19, 515-523. https://doi.org/10.1016/j.tics.2015.07.001

Deckers, K., Köhler, S., Ngandu, T., Antikainen, R., Laatikainen, T., Soininen, H., Strandberg, T., Verhey, F., Kivipelto, M., Solomon, A., 2021. Quantifying dementia prevention potential in the FINGER randomized controlled trial using the LIBRA prevention index. 
Alzheimers Dement 17, 1205-1212. https://doi.org/10.1002/alz.12281

Dimidjian, S., Segal, Z.V., 2015. Prospects for a Clinical Science of Mindfulness-Based Interventions. American Psychologist.

Donald, J.N., Sahdra, B.K., Van Zanden, B., Duineveld, J.J., Atkins, P.W.B., Marshall, S.L., Ciarrochi, J., 2019. Does your mindfulness benefit others? A systematic review and meta-analysis of the link between mindfulness and prosocial behaviour. Br J Psychol 110, 101-125. https://doi.org/10.1111/bjop.12338

Engert, V., Kok, B.E., Papassotiriou, I., Chrousos, G.P., Singer, T., 2017. Specific reduction in cortisol stress reactivity after social but not attention-based mental training. Sci Adv 3, e1700495. https://doi.org/10.1126/sciadv.1700495

Fjell, A.M., Walhovd, K.B., 2010. Structural brain changes in aging: courses, causes and cognitive consequences. Rev Neurosci 21, 187-221. https://doi.org/10.1515/revneuro.2010.21.3.187

Fountain-Zaragoza, S., Prakash, R.S., 2017. Mindfulness Training for Healthy Aging: Impact on Attention, Well-Being, and Inflammation. Front. Aging Neurosci. 9. https://doi.org/10.3389/fnagi.2017.00011

Fox, K.C.R., Dixon, M.L., Nijeboer, S., Girn, M., Floman, J.L., Lifshitz, M., Ellamil, M., Sedlmeier, P., Christoff, K., 2016. Functional neuroanatomy of meditation: A review and meta-analysis of 78 functional neuroimaging investigations. Neurosci \& Biobehav Rev 65, 208-228. https://doi.org/10.1016/j.neubiorev.2016.03.021

Fox, K.C.R., Nijeboer, S., Dixon, M.L., Floman, J.L., Ellamil, M., Rumak, S.P., Sedlmeier, P., Christoff, K., 2014. Is meditation associated with altered brain structure? A systematic review and meta-analysis of morphometric neuroimaging in meditation practitioners. Neuroscience \& Biobehavioral Reviews 43, 48-73. https://doi.org/10.1016/j.neubiorev.2014.03.016

Fredrickson, B.L., Cohn, M.A., Coffey, K.A., Pek, J., Finkel, S.M., 2008. Open hearts build lives: positive emotions, induced through loving-kindness meditation, build consequential personal resources. J Pers Soc Psychol 95, 1045-1062. https://doi.org/10.1037/a0013262

Friedman, E.M., Ruini, C., Foy, C.R., Jaros, L., Love, G., Ryff, C.D., 2019. Lighten UP! A Community-Based Group Intervention to Promote Eudaimonic Well-Being in Older Adults: A Multi-Site Replication with 6 Month Follow-Up. Clin Gerontol 42, 387-397. https://doi.org/10.1080/07317115.2019.1574944

Gard, T., Hölzel, B.K., Lazar, S.W., 2014. The potential effects of meditation on age-related cognitive decline: a systematic review. Ann. N. Y. Acad. Sci. 1307, 89-103. https://doi.org/10.1111/nyas.12348

Garland, E.L., Kiken, L.G., Faurot, K., Palsson, O., Gaylord, S.A., 2017. Upward Spirals of Mindfulness and Reappraisal: Testing the Mindfulness-to-Meaning Theory with Autoregressive Latent Trajectory Modeling. Cogn Ther Res 41, 381-392. https://doi.org/10.1007/s10608-016-9768-y

Geiger, P.J., Boggero, I.A., Brake, C.A., Caldera, C.A., Combs, H.L., Peters, J.R., Baer, R.A., 2016. Mindfulness-Based Interventions for Older Adults: a Review of the Effects on Physical and Emotional Well-Being. Mindfulness 7, 296-307. https://doi.org/10.1007/s12671-015-0444-1

Graser, J., Stangier, U., 2018. Compassion and Loving-Kindness Meditation: An Overview and Prospects for the Application in Clinical Samples. Harv Rev Psychiatry 26, 201-215. https://doi.org/10.1097/HRP.0000000000000192

Innes, K.E., Selfe, T.K., 2014. Meditation as a Therapeutic Intervention for Adults at Risk for Alzheimerâ $\epsilon^{\mathrm{TM}} \mathrm{s}$ Disease â $\epsilon^{\prime \prime}$ Potential Benefits and Underlying Mechanisms. Frontiers in Psychiatry 5. https://doi.org/10.3389/fpsyt.2014.00040

Kaliman, P., Alvarez-López, M.J., Cosín-Tomás, M., Rosenkranz, M.A., Lutz, A., Davidson, R.J., 
2014. Rapid changes in histone deacetylases and inflammatory gene expression in expert meditators. Psychoneuroendocrinology 40, 96-107.

https://doi.org/10.1016/j.psyneuen.2013.11.004

Kim, B.J., Cho, I.S., Cho, K.I., 2017. Impact of Mindfulness Based Stress Reduction Therapy on Myocardial Function and Endothelial Dysfunction in Female Patients with Microvascular

Angina. J Cardiovasc Ultrasound 25, 118-123. https://doi.org/10.4250/jcu.2017.25.4.118

Kivipelto, M., Solomon, A., Ahtiluoto, S., Ngandu, T., Lehtisalo, J., Antikainen, R., Bäckman, L., Hänninen, T., Jula, A., Laatikainen, T., Lindström, J., Mangialasche, F., Nissinen, A., Paajanen, T., Pajala, S., Peltonen, M., Rauramaa, R., Stigsdotter-Neely, A., Strandberg, T., Tuomilehto, J., Soininen, H., 2013. The Finnish Geriatric Intervention Study to Prevent Cognitive Impairment and Disability (FINGER): Study design and progress.

Alzheimer's \& Dementia 9, 657-665. https://doi.org/10.1016/j.jalz.2012.09.012

Klimecki, O.M., Leiberg, S., Lamm, C., Singer, T., 2013. Functional neural plasticity and associated changes in positive affect after compassion training. Cereb. Cortex 23, 15521561. https://doi.org/10.1093/cercor/bhs 142

Kral, T.R.A., Schuyler, B.S., Mumford, J.A., Rosenkranz, M.A., Lutz, A., Davidson, R.J., 2018. Impact of short- and long-term mindfulness meditation training on amygdala reactivity to emotional stimuli. Neurolmage 181, 301-313. https://doi.org/10.1016/j.neuroimage.2018.07.013

Kurth, F., Cherbuin, N., Luders, E., 2017. Promising Links between Meditation and Reduced (Brain) Aging: An Attempt to Bridge Some Gaps between the Alleged Fountain of Youth and the Youth of the Field. Front. Psychol. 8, 860. https://doi.org/10.3389/fpsyg.2017.00860

Lenze, E.J., Hickman, S., Hershey, T., Wendleton, L., Ly, K., Dixon, D., Doré, P., Wetherell, J.L., 2014. Mindfulness-based stress reduction for older adults with worry symptoms and co-occurring cognitive dysfunction. Int J Geriatr Psychiatry. https://doi.org/10.1002/gps.4086

Luders, E., Cherbuin, N., Gaser, C., 2016. Estimating brain age using high-resolution pattern recognition: Younger brains in long-term meditation practitioners. Neuroimage 134, 508 513. https://doi.org/10.1016/j.neuroimage.2016.04.007

Lutz, A., Brefczynski-Lewis, J., Johnstone, T., Davidson, R.J., 2008. Regulation of the neural circuitry of emotion by compassion meditation: effects of meditative expertise. PLoS ONE 3, e1897. https://doi.org/10.1371/journal.pone.0001897

Lutz, A., Jha, A.P., Dunne, J.D., Saron, C.D., 2015. Investigating the phenomenological matrix of mindfulness-related practices from a neurocognitive perspective. Am Psychol 70, 632-658. https://doi.org/10.1037/a0039585

Lutz, A., Klimecki, O.M., Collette, F., Poisnel, G., Arenaza-Urquijo, E., Marchant, N.L., De La Sayette, V., Rauchs, G., Salmon, E., Vuilleumier, P., Frison, E., Vivien, D., Chételat, G., Medit-Ageing Research Group, 2018. The Age-Well observational study on expert meditators in the Medit-Ageing European project. Alzheimers Dement (N Y) 4, 756-764. https://doi.org/10.1016/j.trci.2018.11.002

Malinowski, P., Shalamanova, L., 2017. Meditation and Cognitive Ageing: the Role of Mindfulness Meditation in Building Cognitive Reserve. J Cogn Enhanc 1, 96-106. https://doi.org/10.1007/s41465-017-0022-7

Marchant, N.L., Howard, R.J., n.d. Cognitive Debt and Alzheimer's disease. Journal of Alzheimer's Disease In Press.

Marchant, N.L., Lovland, L.R., Jones, R., Pichet Binette, A., Gonneaud, J., Arenaza-Urquijo, E.M., Chételat, G., Villeneuve, S., PREVENT-AD Research Group, 2020. Repetitive negative thinking is associated with amyloid, tau, and cognitive decline. Alzheimers Dement 16, 1054-1064. https://doi.org/10.1002/alz.12116

Montero-Odasso, M., Ismail, Z., Livingston, G., 2020. One third of dementia cases can be 
prevented within the next 25 years by tackling risk factors. The case "for" and "against." Alz Res Therapy 12, 1-5. https://doi.org/10.1186/s13195-020-00646-x

Moynihan, J.A., Chapman, B.P., Klorman, R., Krasner, M.S., Duberstein, P.R., Brown, K.W., Talbot, N.L., 2013. Mindfulness-based stress reduction for older adults: effects on executive function, frontal alpha asymmetry and immune function. Neuropsychobiology 68, 34-43. https://doi.org/10.1159/000350949

Ngandu, T., Lehtisalo, J., Solomon, A., Levälahti, E., Ahtiluoto, S., Antikainen, R., Bäckman, L., Hänninen, T., Jula, A., Laatikainen, T., Lindström, J., Mangialasche, F., Paajanen, T., Pajala, S., Peltonen, M., Rauramaa, R., Stigsdotter-Neely, A., Strandberg, T., Tuomilehto, J., Soininen, H., Kivipelto, M., 2015. A 2 year multidomain intervention of diet, exercise, cognitive training, and vascular risk monitoring versus control to prevent cognitive decline in at-risk elderly people (FINGER): a randomised controlled trial. Lancet 385, 2255-2263. https://doi.org/10.1016/S0140-6736(15)60461-5

Norton, S., Matthews, F.E., Barnes, D.E., Yaffe, K., Brayne, C., 2014. Potential for primary prevention of Alzheimer's disease: an analysis of population-based data. Lancet Neurol 13, 788-794. https://doi.org/10.1016/S1474-4422(14)70136-X

Poisnel, G., Arenaza-Urquijo, E., Collette, F., Klimecki, O.M., Marchant, N.L., Wirth, M., de La Sayette, V., Rauchs, G., Salmon, E., Vuilleumier, P., Frison, E., Maillard, A., Vivien, D., Lutz, A., Chételat, G., Medit-Ageing Research Group, 2018. The Age-Well randomized controlled trial of the Medit-Ageing European project: Effect of meditation or foreign language training on brain and mental health in older adults. Alzheimers Dement (N Y) 4, 714-723. https://doi.org/10.1016/j.trci.2018.10.011

Prakash, R., Rastogi, P., Dubey, I., Abhishek, P., Chaudhury, S., Small, B.J., 2012. Long-term concentrative meditation and cognitive performance among older adults. Neuropsychol Dev Cogn B Aging Neuropsychol Cogn 19, 479-494. https://doi.org/10.1080/13825585.2011.630932

Quintana-Hernández, D.J., Miró-Barrachina, M.T., Ibáñez-Fernández, I.J., Pino, A.S., QuintanaMontesdeoca, M.P., Rodríguez-de Vera, B., Morales-Casanova, D., Pérez-Vieitez, M. del C., Rodríguez-García, J., Bravo-Caraduje, N., 2015. Mindfulness in the Maintenance of Cognitive Capacities in Alzheimer's Disease: A Randomized Clinical Trial. JAD 50, 217-232. https://doi.org/10.3233/JAD-143009

Richard, E., Van den Heuvel, E., Moll van Charante, E.P., Achthoven, L., Vermeulen, M., Bindels, P.J., Van Gool, W.A., 2009. Prevention of dementia by intensive vascular care (PreDIVA): a cluster-randomized trial in progress. Alzheimer Dis Assoc Disord 23, 198204. https://doi.org/10.1097/WAD.0b013e31819783a4

Ryff, C.D., Heller, A.S., Schaefer, S.M., van Reekum, C., Davidson, R.J., 2016. Purposeful Engagement, Healthy Aging, and the Brain. Curr Behav Neurosci Rep 3, 318-327. https://doi.org/10.1007/s40473-016-0096-z

Scarmeas, N., Stern, Y., 2003. Cognitive reserve and lifestyle. J Clin Exp Neuropsychol 25, 625-633. https://doi.org/10.1076/jcen.25.5.625.14576

Schuman-Olivier, Z., Trombka, M., Lovas, D.A., Brewer, J.A., Vago, D.R., Gawande, R., Dunne, J.P., Lazar, S.W., Loucks, E.B., Fulwiler, C., 2020. Mindfulness and Behavior Change. Harv Rev Psychiatry 28, 371-394. https://doi.org/10.1097/HRP.0000000000000277

Schutte, N.S., Malouff, J.M., 2014. A meta-analytic review of the effects of mindfulness meditation on telomerase activity. Psychoneuroendocrinology 42, 45-48. https://doi.org/10.1016/j.psyneuen.2013.12.017

Singer, T., Klimecki, O.M., 2014. Empathy and compassion. Curr. Biol. 24, R875-R878. https://doi.org/10.1016/j.cub.2014.06.054

Smart, C.M., Segalowitz, S.J., Mulligan, B.P., Koudys, J., Gawryluk, J.R., 2016. Mindfulness Training for Older Adults with Subjective Cognitive Decline: Results from a Pilot Randomized Controlled Trial. JAD 52, 757-774. https://doi.org/10.3233/JAD-150992 
Tang, Y.-Y., Hölzel, B.K., Posner, M.I., 2015. The neuroscience of mindfulness meditation. Nat. Rev. Neurosci. 16, 213-225. https://doi.org/10.1038/nrn3916

Teipel, S., Drzezga, A., Grothe, M.J., Barthel, H., Chételat, G., Schuff, N., Skudlarski, P., Cavedo, E., Frisoni, G.B., Hoffmann, W., Thyrian, J.R., Fox, C., Minoshima, S., Sabri, O., Fellgiebel, A., 2015. Multimodal imaging in Alzheimer's disease: validity and usefulness for early detection. Lancet Neurol 14, 1037-1053. https://doi.org/10.1016/S1474-4422(15)00093-9

Valk, S.L., Bernhardt, B.C., Trautwein, F.-M., Böckler, A., Kanske, P., Guizard, N., Collins, D.L., Singer, T., 2017. Structural plasticity of the social brain: Differential change after socioaffective and cognitive mental training. Sci Adv 3, e1700489. https://doi.org/10.1126/sciadv. 1700489

Victorson, D., Kentor, M., Maletich, C., Lawton, R.C., Kaufman, V.H., Borrero, M., Languido, L., Lewett, K., Pancoe, H., Berkowitz, C., 2015. Mindfulness Meditation to Promote Wellness and Manage Chronic Disease: A Systematic Review and Meta-Analysis of Mindfulness-Based Randomized Controlled Trials Relevant to Lifestyle Medicine. American Journal of Lifestyle Medicine 9, 185-211. https://doi.org/10.1177/1559827614537789

Yegambaram, M., Manivannan, B., Beach, T.G., Halden, R.U., 2015. Role of environmental contaminants in the etiology of Alzheimer's disease: a review. Curr Alzheimer Res 12, 116-146. https://doi.org/10.2174/1567205012666150204121719

Zellner Keller, B., Singh, N.N., Winton, A.S.W., 2014. Mindfulness-Based Cognitive Approach for Seniors (MBCAS): Program Development and Implementation. Mindfulness (N Y) 5, 453-459. https://doi.org/10.1007/s12671-013-0262-2

Zufferey, V., Donati, A., Popp, J., Meuli, R., Rossier, J., Frackowiak, R., Draganski, B., von Gunten, A., Kherif, F., 2017. Neuroticism, depression, and anxiety traits exacerbate the state of cognitive impairment and hippocampal vulnerability to Alzheimer's disease. Alzheimers Dement (Amst) 7, 107-114. https://doi.org/10.1016/j.dadm.2017.05.002

\section{Figure 1}

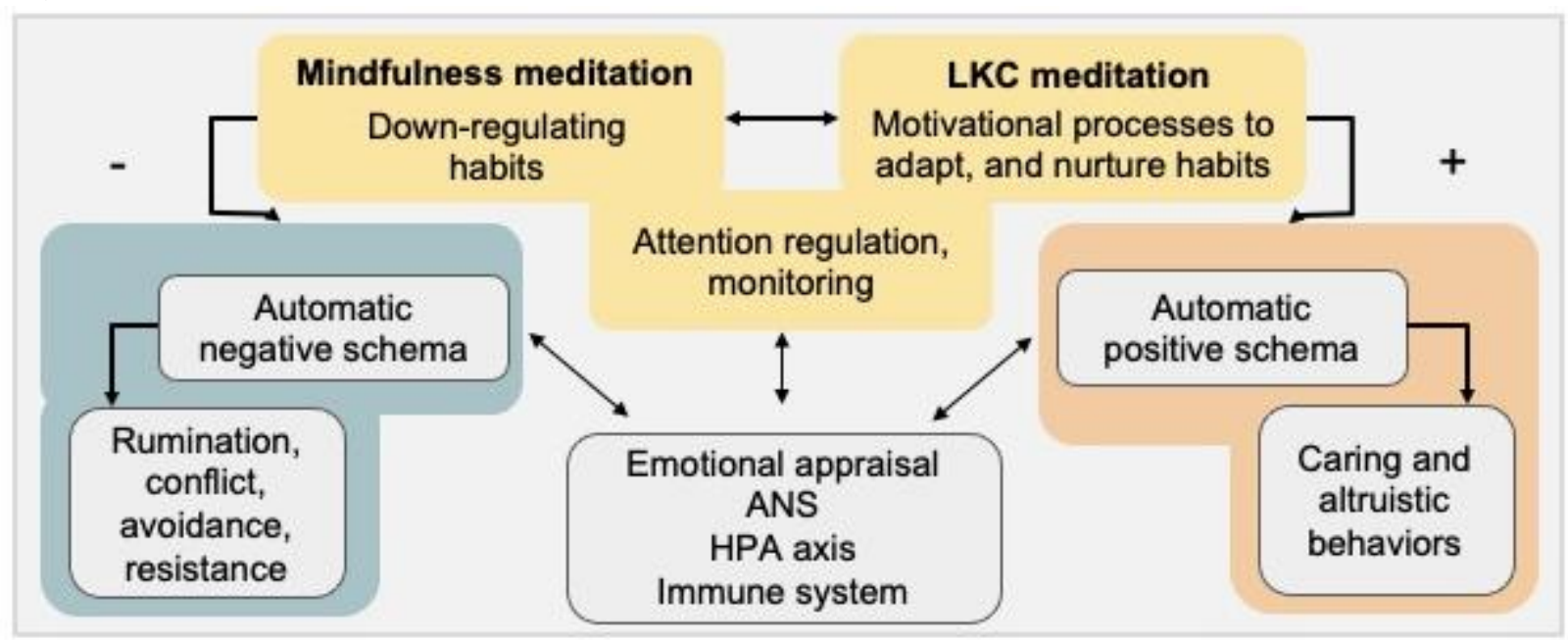

Figure 2 


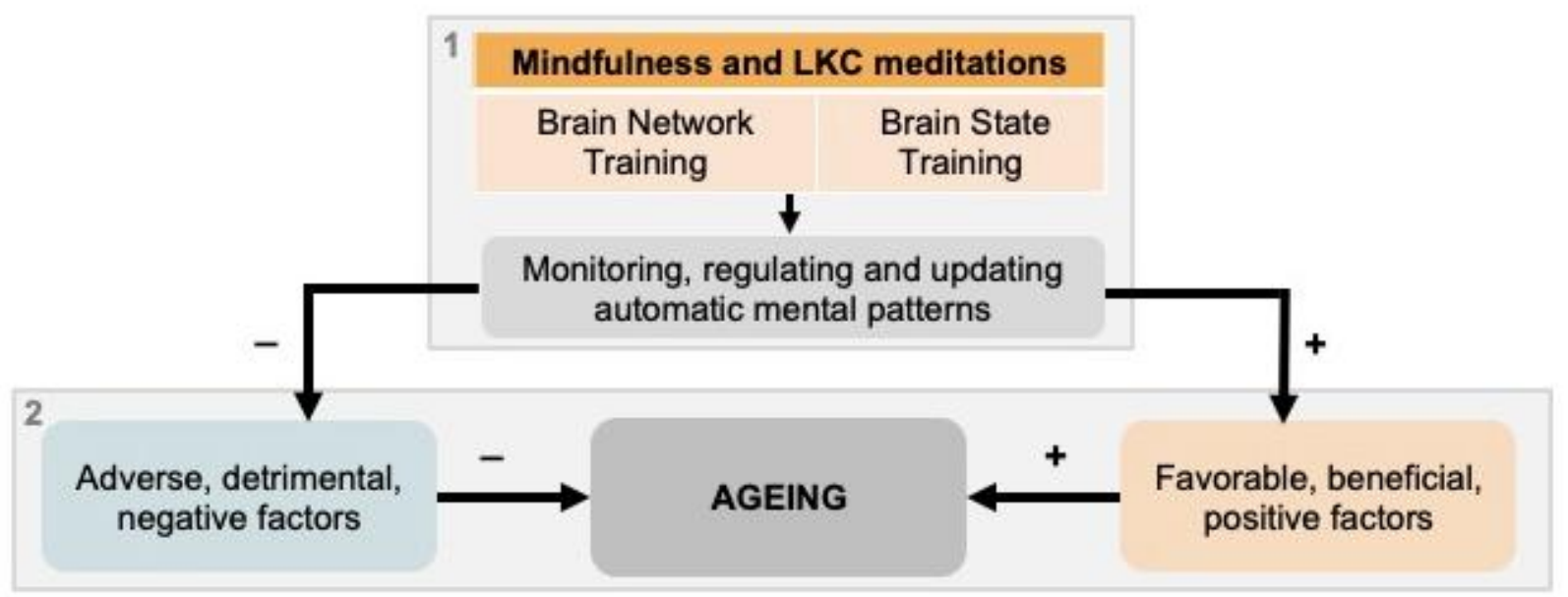

Figure 3 


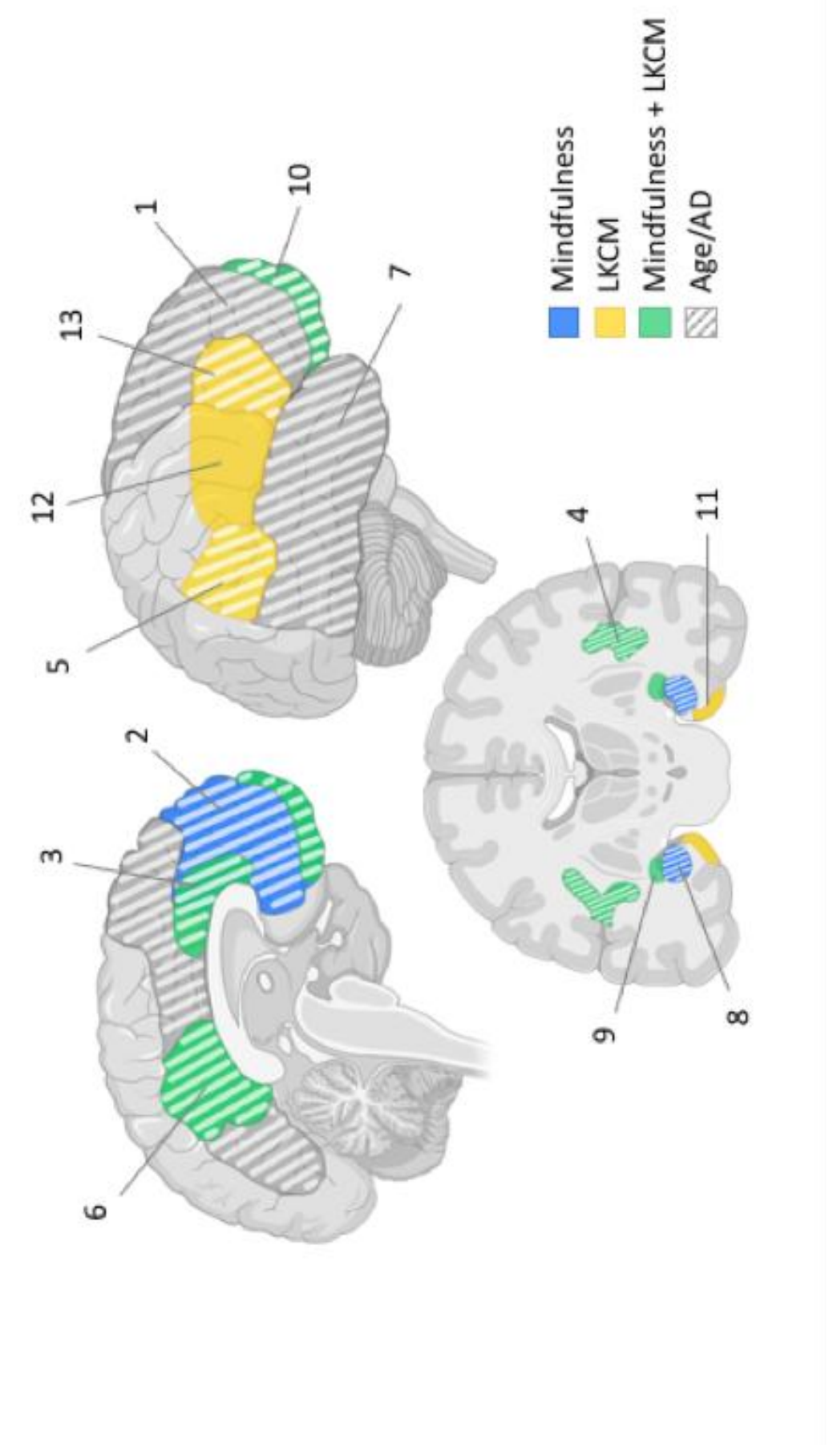

